\title{
Light-induced reflectivity transients in black-Si nanoneedles
}

\author{
P. Ščajev, ${ }^{1, *}$ T. Malinauskas, ${ }^{1}$ G. Seniutinas,${ }^{2,3}$ M. D. Arnold, ${ }^{4}$ A. Gentle, ${ }^{4}$ I. Aharonovich, ${ }^{4}$ \\ G. Gervinskas, ${ }^{2,3}$ P. Michaux, ${ }^{2}$ J. S. Hartley, ${ }^{2}$ E. L. H. Mayes, ${ }^{5}$ P. R. Stoddart, ${ }^{2}$ and S. Juodkazis ${ }^{2,3}, \dagger$ \\ ${ }^{1}$ Institute of Applied Research, Vilnius University, \\ Sauletekio Avenue 9, Bldg. III, LT-10222 Vilnius, Lithuania \\ ${ }^{2}$ Faculty of Science, Engineering and Technology, \\ Swinburne University of Technology, Hawthorn, VIC 3122, Australia \\ ${ }^{3}$ Melbourne Centre for Nanofabrication, ANFF, 151 Wellington Road, Clayton, VIC 3168, Australia \\ 4 School of Physics and Advanced Materials, University of Technology Sydney, 15 Broadway, Ultimo, NSW 2007, Australia \\ ${ }^{5}$ School of Applied Sciences, RMIT University, GPO Box 2476 V, Melbourne, VIC 3001, Australia
}

(Dated: August 25, 2015)

\begin{abstract}
The change in reflectivity of black-Si (b-Si) upon optical excitation was measured by the pumpprobe technique using picosecond laser pulses at 532 (pump) and $1064 \mathrm{~nm}$ (probe) wavelengths. The specular reflection from the random pattern of plasma-etched b-Si nano-needles was dominated by the photo-excited free-carrier contribution to the reflectivity. The kinetics of the reflectivity were found to be consistent with surface structural and chemical analysis, performed by scanning and transmission electron microscopy, and spectroscopic ellipsometry. The surface recombination velocity on the b-Si needles was estimated to be $\sim 10^{2} \mathrm{~cm} / \mathrm{s}$. Metalization of b-Si led to much faster recombination and alteration of reflectivity. The reflectivity spectra of random b-Si surfaces with different needle lengths was modeled by a multi-step refractive index profile in the Drude formalism. The dip in the reflectivity spectra and the sign reversal in the differential reflectivity signal at certain b-Si needle sizes is explained by the model.
\end{abstract}

PACS numbers: 81.07.-b

\section{INTRODUCTION}

The fabrication and replication of nano-textured surfaces is a fast growing area of research, especially in the biomedical field where surface structures can have a significant effect on the functions of cells and membrane receptors ${ }^{1}$. When coated with thin films or nanoparticles of plasmonic metals, nanostructured surfaces are also widely employed as substrates for surface-enhanced Raman scattering (SERS) ${ }^{2,3}$. In this context, black-Si (b-Si) has attracted increasing attention, as the surface area, aspect ratio of the nano-needles, surface hydrophobicity, changes in the surface recombination rate and antireflection properties are all very useful parameters for energy and sensing applications ${ }^{2,4,5}$.

The surface structure and properties of b-Si can be controlled via the plasma- or chemical- etching conditions used in fabrication. Black-Si solar cells with high efficiency $(>18 \%)$ have been achieved ${ }^{6}$, even without an antireflection coating. The tapered needles create a gradual change in refractive index, which reduces the reflectivity of a step-like change in refractive index, as demonstrated in the case of glass ${ }^{7}$ and in some naturally nanostructured surfaces ${ }^{8}$. The porosity of Si can also be controlled by electrochemical etching to change the refractive index and surface passivation ${ }^{9}$. Future solar cells with light harvesting efficiencies of over $90 \%$ and a Si thickness of just micrometers have been designed using slanted conical holes ${ }^{10}$.

In all of these light harvesting and sensor applications, structural defects and chemical contamination on the surface of plasma- (or chemically-) etched surfaces have to be understood and controlled. This study explores the effect of plasma etching on light localization, absorption and thermal properties, using a picosecond pump-probe spectroscopy technique to investigate the photoinduced reflectivity of b-Si. Black-Si with different morphologies was prepared by plasma etching. The unperturbed reflectivity of random b-Si surfaces was modeled by a multistep refractive index profile and matched to experimental ellipsometry data.

\section{RESULTS}

All of the samples in this study were made by the same plasma etching recipe, but with different etching times. Figure 1 shows SEM images of b-Si etched for $5 \mathrm{~min}$ (a,b) and $25 \mathrm{~min}(\mathrm{c}, \mathrm{d})$. The separation between the bSi needles followed a diffusional dependence on etching time, with an average separation of $388 \pm 20 \mathrm{~nm}$ for 5 min and $842 \pm 30 \mathrm{~nm}$ for $25 \mathrm{~min}$ etch times (Fig. 1). For Batch No. 1, the separation was $\sim 300 \mathrm{~nm}$ and the pillars had a pyramidal shape of height $\sim 500 \mathrm{~nm}$. Batches 12 had comparable pillar heights ${ }^{11}$, while Batch 3 has a pillar height of $4 \mu \mathrm{m}$ as evident from Fig. 1(d).

Figure 1(e) shows the surface chemical composition and morphology of a sample from Batch No. 3. X-ray photo-electron spectroscopy (XPS) revealed no significant differences between samples from Batches 1-3. Surface contamination by fluorine and oxygen was present due to the mixture of $\mathrm{SF}_{6}$ and $\mathrm{O}_{2}$ used for plasma etching. The tips of the needles were crystalline silicon without any amorphization or distinct oxide layer. This is an 

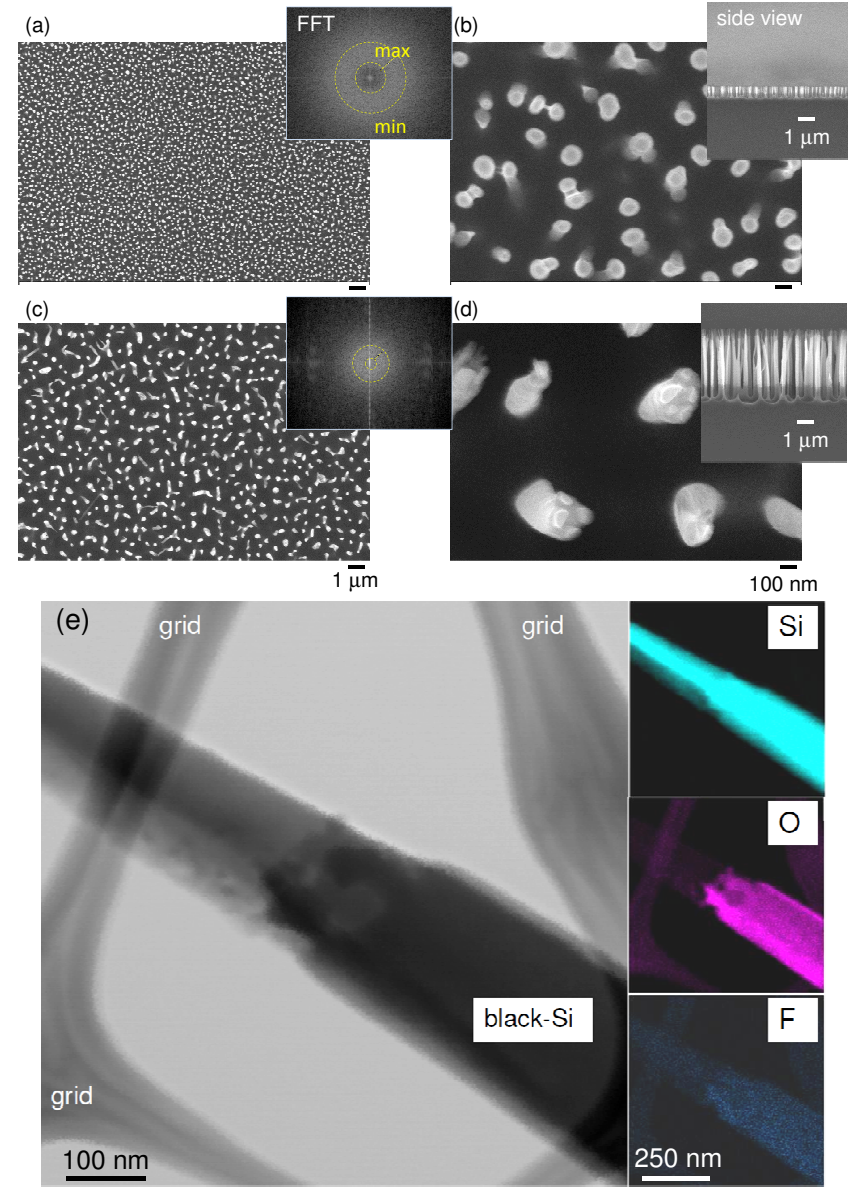

FIG. 1: Top view SEM images of b-Si etched for 5 min (a,b) and 25 min (c,d) (Batches 2 and 3, respectively). Insets in $(\mathrm{a}, \mathrm{c})$ are fast Fourier transform (FFT) images with the inner $(\max )$ and outer $(\min )$ dashed-circles corresponding to $692-$ $261 \mathrm{~nm}$ (max-min) in (a) and 1635-573 $\mathrm{nm}$ in (c). Insets in (b,d) are side-view SEM images. (e) Elemental mapping was conducted using scanning-TEM mode and an EDS detector (Batch No. 3).

important point, as it allowed the optical response to be modeled without having to account for changes in surface composition.

The reflectivity of the samples was measured with a bifurcated optical fiber spectrometer, where white light illumination was delivered via the central fiber and the back-reflected (and scattered) light was collected by satellite fibers. As shown in Fig. 2, a recognizable reflectivity dip was observed and its spectral position and amplitude were dependent on the structure of the b-Si.

Although the reflection from the b-Si was low ( $1-2 \%$ ), it was close to specular. In a pump-probe experiment, specular reflection is not expected when the wavelength of the pump laser is smaller than the spacing between the needles. However, the reflectivity measured by pump-probe at a distance tens-of-centimeters from the b-Si surface was comparable to that measured with

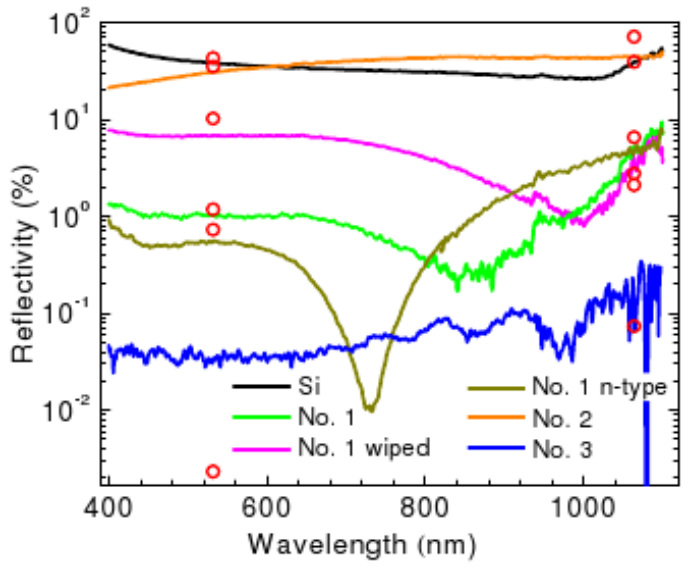

FIG. 2: Reflection spectra of different samples (see Experimental Section for details) measured at normal incidence by a bifurcated optical fiber spectrometer. The minima at 700$1000 \mathrm{~nm}$ correspond to reflection from a random structure of pillars $\sim 0.5 \mu \mathrm{m}$ high. The "wiped" sample indicates that the needles were mechanically abraded; the n-type b-Si was Asdoped with $\langle 100\rangle$ orientation and resistivity $R \leq 0.005 \Omega / \mathrm{cm}$ made by the recipe of Batch No. 1 . The Au coating was over $200 \mathrm{~nm}$ thick for the reflective mirror-like sample and was $50 \mathrm{~nm}$ for the least reflective Batch No. 3. The circles show the reflectivity measured by pump-probe experiments on the respective samples; the largest mismatch was for Batch No. 3.

the bifurcated fiber spectrometer directly at the surface. Hence, reflectivity (transmission) measurements can be carried out under laser illumination, providing a contactless optical measurement of excited carrier dynamics in the sub-surface regions of the needles.

The differential reflectivity, $D R(t)$, is defined in terms of the time-dependent sample reflectivity $R(t)$ and the equilibrium reflectivity $R_{0}$ (see Eq. 1$)^{12}$. The differential reflectivity kinetics are dependent on the decay of the carrier density $\Delta N$ within a depth $d_{a b s} \sim \lambda_{e x} / 4 \pi k_{e x} \simeq$ $2.1 \mu \mathrm{m}$; here the excitation $\lambda_{e x}=532 \mathrm{~nm}$ and $k_{e x} \simeq$ 0.02 are the pump wavelength and imaginary part of the refractive index of $\mathrm{Si}$, respectively ${ }^{13}$.

The longer wavelength probe is absorbed over a much longer distance, and thus the probe absorption is usually unimportant compared with the short scale refractive index changes due to interfaces and carrier profile. In b-Si the fraction of $\mathrm{Si}$ is reduced, making the effective depths longer ${ }^{12,14}$. The refractive index of Si for the probe beam is $n_{p r}=3.5$ and $\omega_{p r}=2 \pi \nu_{p r}$ is the cyclic frequency of the probe. The backside of the b-Si samples was not polished, thus excluding any contribution of backside reflection to the measured $D R$ signal (the scattered reflected light was blocked by an auxiliary aperture).

The proportionality factor $n_{e h}$ represents the refractive index change per electron-hole pair in a unit volume. The Drude formalism ${ }^{15}$ allows $n_{e h}$ to be obtained according to Eq. 2, where the electron-hole mass in $\mathrm{Si}$ is $m_{e h}=0.166 m_{0}$ and depends on the electron and hole 


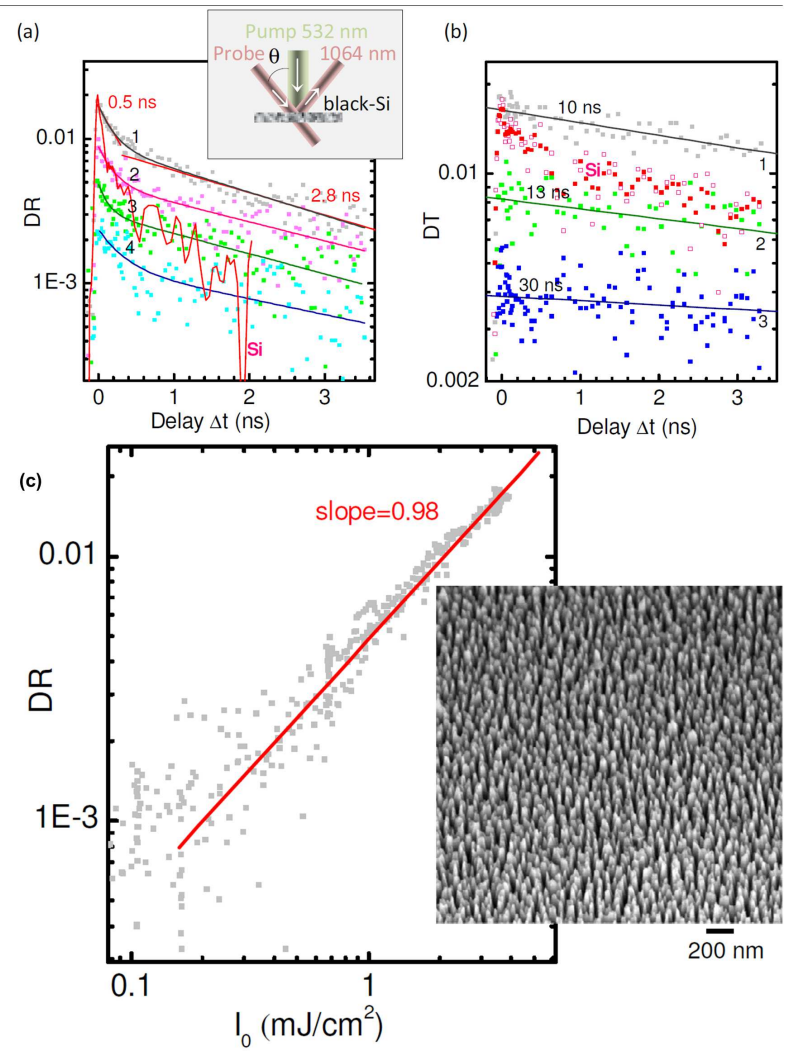

FIG. 3: Photo-induced changes of (a) reflection $D R=\Delta R / R$ and (b) transmission $D T=\Delta T / T$ for Batch No. 1 in a pumpprobe experiment. Inset in (a): measurement geometry where the angle of incidence $\theta=23^{\circ}$. Intensities in (a): $3.5 \mathrm{~mJ} / \mathrm{cm}^{2}$ (1), 1.7 (2), 0.9 (3), 0.4 (4), and in (b): 2 (1), 1 (2), 0.5 (3). Si was measured as a reference (red markers) at $1.8 \mathrm{~mJ} / \mathrm{cm}^{2}$ excitation. The fitted (solid) curves are (a) bi-exponential and (b) single exponential fits. Note that the sign of $D R$ for $\mathrm{Si}$ in (a) is reversed. (c) Power dependence of the reflection peak $D R$ for Batch No. 1. The reflectivity of b-Si increased with excitation $(\Delta R>0)$ while it decreased $(\Delta R<0)$ for $\mathrm{Si}$. Inset shows a slanted-view SEM image of the sample.

conductivity masses, $m_{e}=0.26 m_{0}$ and $m_{h}=0.46 m_{0}{ }^{16}$, respectively, while $\varepsilon_{0}$ is the vacuum dielectric constant and $m_{0}$ is the electron mass ${ }^{12}$ :

$$
\begin{gathered}
D R(t)=\frac{R(t)-R_{0}}{R_{0}} \approx-\frac{4}{n_{p r}^{2}-1}\left|n_{e h}\right| \Delta N\left(z=d_{o b s}, t\right), \\
n_{e h}=-\frac{e^{2}}{2 n_{p r} \varepsilon_{0} m_{e h}^{*} \omega_{p r}^{2}}, \quad \frac{1}{m_{e h}^{*}}=\frac{1}{m_{e}^{*}}+\frac{1}{m_{h}^{*}} .
\end{gathered}
$$

Note that $n_{p r}$ is the refractive index for the probe beam, and $d_{o b s} \sim \lambda_{p r} / 4 \pi n_{p r}$ is the effective probe observation depth at a single interface ${ }^{12}$. The factor $n_{e h}$ was calculated as $9.3 \times 10^{-22} \mathrm{~cm}^{3}$ for Si. The differential transmission is defined as

$$
D T(t)=\ln \left(T_{0} / T(t)\right) \approx \int_{0}^{d} \Delta \alpha_{p r}(t, z) d z,
$$

where $z$ is the depth coordinate. Note that these equations are applicable for bulk Si, while the absorption in the needles and reflection from the Si substrate must be considered for b-Si (for details see discussion later in this Section).

The refractive index changes due to free carriers $\Delta n_{c}=$ $n_{e h} \Delta N$ are much higher than those due to temperature changes $\Delta T$ induced by excitation, $\Delta n_{T}=n_{T} \Delta T$, where $n_{T}=2.2 \times 10^{-4} \mathrm{~K}^{-1}$ is the thermooptic coefficient of Si at room temperature ${ }^{17}$. The ratio $-\Delta n_{c} / \Delta n_{T}$ can be calculated according to $\left(n_{e h} / \hbar \omega_{e x}\right) /\left(n_{T} / \rho C_{p}\right)^{18}$, where $C_{p}=$ $0.71 \mathrm{~J} / \mathrm{gK}$ is the heat capacity ${ }^{19}$ and $\rho=2.33 \mathrm{~g} / \mathrm{cm}^{3}$ is the density of Si. The estimated ratio of $\sim 19$ indicates that refractive index changes due to sample heating by photo-excitation can be disregarded.

The time-dependent differential transmission of the probe beam (DT, Eq. 3) is obtained by integrating the excess carrier density over the sample thickness $d$ at different delay times with respect to the pump beam ${ }^{20}$. Here $T_{0}$ and $T(t)$ are the transmission of the probe beam when the pump is off and on, respectively. The amplitude of $D T$ is defined by the magnitude of the induced absorption change for the probe wavelength in Eq. 3, namely $\Delta \alpha_{p r}(t, z)=\Delta \alpha_{F C A}=\sigma_{e} \Delta n+\sigma_{h} \Delta p \sim \sigma_{e h} \Delta N$, which consists of the free carrier absorption coefficient $\Delta \alpha_{F C A}$ due to the excess concentration of electrons $\Delta n$ and holes $\Delta p$, respectively; $\sigma_{e}$ and $\sigma_{h}$ are the absorption cross sections for the free electrons and holes, respectively, while $\sigma_{e h}$ is the total free carrier absorption cross section ${ }^{20}$ at concentration $\Delta N$. The free carrier absorption modifies the imaginary part of the refractive index by ${ }^{21} \Delta k=$ $\Delta \alpha_{p r} \lambda / 4 \pi$, which reduces to $\Delta k=\sigma_{e h}(\lambda) \Delta N \lambda / 4 \pi$. The dependence of $\Delta k$ on wavelength $\lambda$ can be approximated by $^{22} \sigma_{e h}(\lambda) \sim(3.5 \pm 0.5) \times 10^{-18} \times(\lambda[\mu \mathrm{m}])^{2.8}$ in the interval $0.86-2.0 \mu \mathrm{m}$. The peak $D T$ signal is defined by the relationship $D T=\sigma_{e h} \Delta N_{0} / \alpha_{e x}$.

Batch 1 exhibited the best optical properties: the lowest surface recombination velocity, $S$, at the air-needle interface and the best absorption enhancement in Si i.e. the $D R$ signal was $30 \%$ larger than in bulk Si. The darkest b-Si sample with the highest aspect ratio needles (Batch No. 3) showed no probe transmission after a $50 \mathrm{~nm} \mathrm{Pd}$ coating, most probably due to large $S$ and $k$ the imaginary part of the refractive index.

The reflection minimum at $\lambda_{\min }$, observed in Fig. 2, can be explained by thin film interference between the top of the b-Si and the b-Si|Si interface. Thus reducing the needle length by mechanical abrasion shifted the minimum by $150 \mathrm{~nm}$ ("wiped" sample). A $200 \mathrm{~nm}$ Au coating provided very strong reflectivity, indicating that a reflective $\mathrm{Au}$ layer was formed over the tips of the densely packed needles (separation $\sim 300 \mathrm{~nm}$, see Fig. 1). In con- 


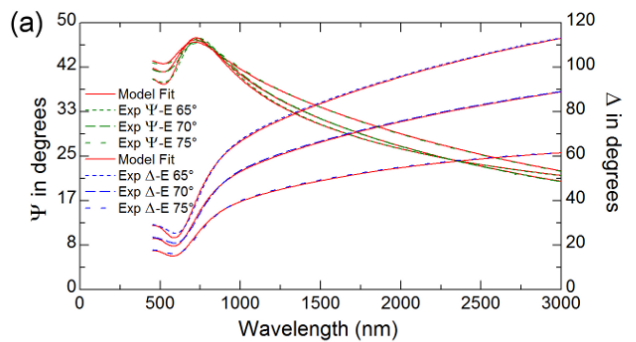

(c) Depth Profile of Optical Constants at $532 \mathrm{~nm}$

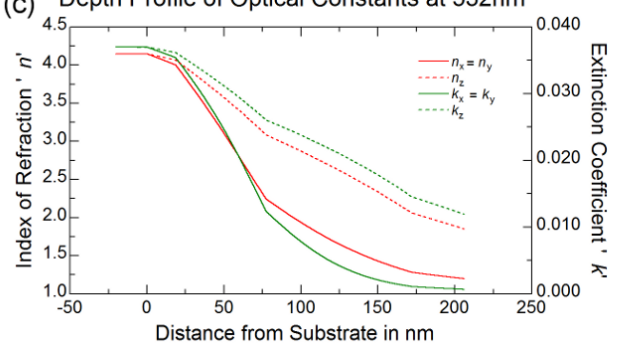

(b)

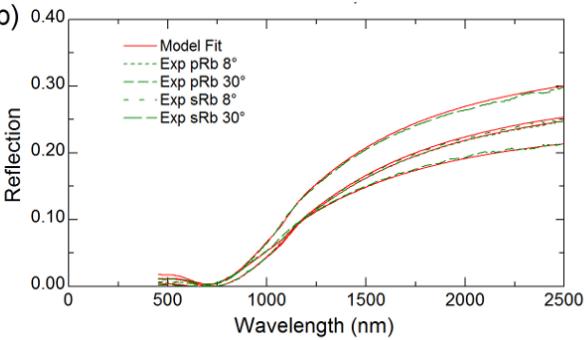

(d) Depth Profile of Optical Constants at $1064 \mathrm{~nm}$

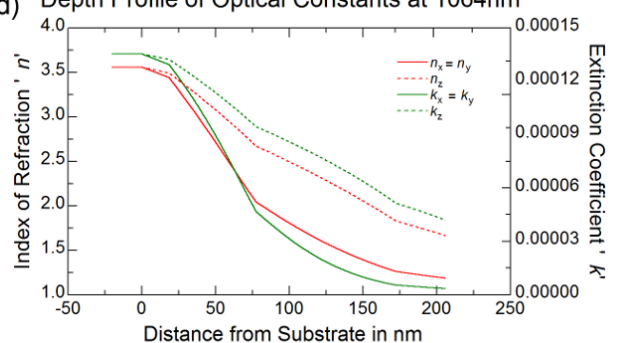

FIG. 4: Ellipsometry measurements and model results for a typical b-Si sample consisting of pyramidal needles. The reflectance ratio for the E-field of $s-$ and $p$-polarizations was measured as $\frac{r_{p}}{r_{s}} \equiv \tan (\Psi) e^{1 \Delta}$ over a wide range of wavelengths, where tan $\Psi$ is the reflection amplitude and $\Delta$ is the phase shift. (a) $\Psi$ and $\Delta$ at different angles of incidence. (b) Simulated and experimental polarized reflectance spectra. (c,d) Geometrical depth profiles of refractive index $(n+1 k)$ for which the best fit was achieved at $532 \mathrm{~nm}$ and $1064 \mathrm{~nm}$, respectively.

trast, a strongly reduced reflectivity was observed for the $50 \mathrm{~nm}$ coating (Batch 3). This reduction may be related to the impact of long $(\sim 4 \mu \mathrm{m})$ and more separated $(\sim 800 \mathrm{~nm})$ needles (Fig. 1d).

The $D R$ decays in Fig. 3 are non-exponential and weakly excitation dependent, indicating that surface and nonlinear recombination may have an impact ${ }^{14}$. An upper bound of the surface recombination velocity in bSi can be estimated, assuming that it occurs predominantly on the cylindrical needle surfaces. According to the relationship ${ }^{23} \tau_{S}=0.282 d_{n} / S$, with needle diameter $d_{n} \sim 200 \mathrm{~nm}$ and $\tau_{S}>500 \mathrm{ps}$, a surface recombination velocity of $S<113 \mathrm{~cm} / \mathrm{s}$ was calculated on the needle surface. Such a low value is typical for Si passivated by native dielectric $\mathrm{SiO}_{2}{ }^{24}$. A non-exponential decay may be caused by random variations in the $d_{n}$ and $S$ values. Therefore faster $D R$ decays indicate larger $S$ values. On metal-coated needles (Batch 2 in Fig. 3), the lifetime was found to be less than $\sim 10 \mathrm{ps}$, which leads to $S>10^{4} \mathrm{~cm} / \mathrm{s}$, which is typical for Pd-metalised $\mathrm{Si}^{25}$. Note that noble metals such as $\mathrm{Pt}, \mathrm{Pd}$ and $\mathrm{Au}$ are known to reduce the recombination lifetime in $\mathrm{Si}^{26}$.

The DT decay rate (Fig. 3(b)) increases with increasing excitation intensity, indicating the presence of nonlinear recombination. The Auger coefficient in highly excited silicon is $C=3 \times 10^{-31} \mathrm{~cm}^{6} / \mathrm{s}$ in the 77 $400 \mathrm{~K}$ temperature range ${ }^{27}$. The $1.8 \mathrm{~mJ} / \mathrm{cm}^{2}$ excitation provides $\Delta N_{0}=4.3 \times 10^{19} \mathrm{~cm}^{-3}$ and an Auger lifetime of $\tau_{A u g}=3 /\left(C \Delta N_{0}^{2}\right)=6.7 \mathrm{~ns}$, which is close to the experimental results. Here, the factor of $3 \mathrm{ac}-$ counts for an inhomogeneous distribution of excited car- riers with depth ${ }^{14}$. The differential transmission peaks at $D T \simeq 0.018$ for $1.8 \mathrm{~mJ} / \mathrm{cm}^{2}$ excitation, according to $D T=\sigma_{e h}(1064 \mathrm{~nm}) \Delta N_{0} / \alpha_{e x}$ and provided that $\sigma_{e h}(1064 \mathrm{~nm}) \simeq 3.7 \times 10^{-18} \mathrm{~cm}^{2}$, which is close to the $4.2 \times 10^{-18} \mathrm{~cm}^{2}$ reported in the literature ${ }^{22}$. It is noteworthy that $D T$ also probes the underlying Si layer as the excitation depth exceeds the b-Si thickness (in b-Si the carrier density should be homogeneous with $\Delta N_{0}$ as the Si needles fill only a small fraction, $\sim 10 \%$, of the b-Si volume). Thus $D R$ is more advantageous for b-Si studies than $D T$.

For comparison, the $D R$ decay was measured in bare Si. The decay rate was faster in bare $\mathrm{Si}$ than in Batch No. 1, indicating that Si has a higher surface recombination velocity than a b-Si covered $\mathrm{Si}$ surface. The curve on bare Si showed $D R=0.02$ at $3.5 \mathrm{~mJ} / \mathrm{cm}^{2}$ excitation. The measured value is a close match for the theoretical value of $D R=0.025$ from Eq. 1. The power law of $D R$, shown in Fig. 3, follows a linear dependence on the pump intensity, indicating that the absorption is excitation independent.

The $D R$ decays were measured at different temperatures in a nitrogen cell (see Supplementary Information). The decay amplitude becomes larger and the decay is faster at higher temperatures. A doubling of the decay amplitude in the range $300-500 \mathrm{~K}$ correlates well with an increase in the absorption coefficient from $8 \times 10^{3} \mathrm{~cm}^{-1}$ to $1.5 \times 10^{4} \mathrm{~cm}^{-1}$ over the same range ${ }^{15}$. This is expected as $D R \sim \Delta N \sim \alpha$. The reduction in the initial lifetime from $0.6 \mathrm{~ns}$ to $0.15 \mathrm{~ns}$ also contributes to the increased absorption coefficient, as it leads to a doubling of the 
carrier density near the surface, which in turn provides a four-fold reduction in lifetime as $\tau_{A u g} \sim 1 / \Delta N_{0}^{2}$. A density of $\Delta N_{0}=7.5 \times 10^{19} \mathrm{~cm}^{-3}$ at RT with an Auger coefficient $^{27} C=3 \times 10^{-31} \mathrm{~cm}^{6} / \mathrm{s}$ provides a lifetime of $0.59 \mathrm{~ns}$, which is consistent with our results. Additionally, at the highest temperatures $(600 \mathrm{~K})$ surface recombination may increase due to increased carrier thermal energy, which allows the carriers to overcome the surface trap energy barrier on the needle surfaces, and thus leads to a much faster decay tail at $600 \mathrm{~K}$.

Both ellipsometric and polarised angular reflectance data was used to retrieve the geometrical profile of the random tapered structures via a spectrally wide best fit. Figure 4 shows the measured and modeled ellipsometry (a) and reflectance (b) results for a typical b-Si sample. The structure was modeled using a graded bi-axial Bruggerman effective medium model ${ }^{28}$ using the WVASE ellipsometric modelling software. The tapering of the structure can be seen in Fig. 4(c,d) with the refractive index going from that of bulk silicon at the base and reducing as it reaches the peaks. In order to get a good fit to the reflectance data at the longer wavelengths below the silicon bandgap, a small amount of backside reflection (4\%) was included in the model, consistent with a somewhat wider collection angle than other measurements. For the purposes of initial fitting the graded profile was fitted with 5 nodes, each with 10 steps interpolated between each node. Starting values for the b-Si profile were based on the SEM profile, i.e. needles on top of pyramids (Fig. 1).

The effect of sample parameters on the pump-probe differential reflectance was explored using a detailed thinfilm model. Free-carrier material properties were coupled with the pump intensity profile in the effective-medium stack, with a maximum external pump of $3.5 \mathrm{~mJ} / \mathrm{cm}^{2}$ at $532 \mathrm{~nm}$ (see the Experimental Section for details). Similarly to Eqs. 1 and 2, this model predicts that a negative change in reflectance is possible and will be highest at long wavelengths due to a stronger free-carrier effect and increased absolute reflectance. However, unlike the earlier equations, the detailed model also predicts the possibility of a positive change in reflectance due to spectral shifts.

Representative results are presented in Fig. 5, for a linear conical profile, since this is the simplest graded profile and a reasonable first approximation to the observed structure. Overall, the results show that (for a fixed probe wavelength) the sign of the differential reflectance oscillates with black-Si thickness, in particular changing from negative at small thicknesses, to positive and then to negative again near a dip in absolute reflectance. There are two effects contributing to this response, both of them due to photocarriers reducing the refractive index. Reduced single-interface reflection, as predicted by Eq. 1, affects all samples and is the most important effect in bulk Si. In black Si layers, there is a superimposed reflection oscillation, which on photoexcitation is blue-shifted by reduced optical path length.
This shifted oscillation provides a plausible explanation for the pump-probe results, since Batch 1 (p-type) had a reflection minimum at around $900 \mathrm{~nm}$ with positive differential reflectance at $1064 \mathrm{~nm}$, whereas other samples with different minima had small or negative differential reflectance. More pinched graded profiles (broader bases and needle-like tips) stretch the differential oscillations to longer wavelengths and broaden the absolute reflection dips for a given total thickness, which may refine the quantitative match to the experimental results, however the fundamental behavior remains the same.

In addition, the effect of the doping concentration was found to be minimal over practical resistivity ranges, assuming that backside reflection is ignored. Backside reflection can significantly alter the total reflectance, so we have carefully considered this assumption. Photoexcitation may change the material absorbance enough to switch the backside reflection on or off, even though the carriers are only concentrated within the pump absorption length (about $1 \mu \mathrm{m}$ in depth). At $1064 \mathrm{~nm}$ and 3.5 $\mathrm{mJ} / \mathrm{cm}^{2}$, the effect is negligible below $0.002 \Omega . \mathrm{cm}$, and has largely saturated by about $0.2 \Omega . \mathrm{cm}$, in line with the change in mobility. For bulk Si samples where the frontside reflection is very high, the effect is only about $2 \%$ of the differential signal, but for low reflecting samples with sufficient resistivity the effect could be an important contributor. However, it is reasonable to ignore backside reflection here because the rough backside surface scatters this contribution and it is largely excluded by the specular collection employed. This is confirmed by the observed pump-probe intensity dependence, which is linear in contrast to the non-linear backside dependence. The low resistivity used in Fig. 5 would suppress backside reflection and the results are reasonably representative in other respects.

\section{CONCLUSIONS}

Black-Si was produced on Si surfaces by reactive ion etching with different needle heights from 0.2 to $4 \mu \mathrm{m}$. Controlling the process conditions allows b-Si to be obtained with low reflectivity and high carrier generation efficiency in the underlying $\mathrm{Si}$, providing a simple means of producing an antireflection coating on Si. Metalization of b-Si and modification of the needle length allows either high or ultra low $0.04 \%$ reflectivity to be obtained. The low reflectivity material is promising for thermal sensor applications ${ }^{4}$.

Differential reflectivity decay measurements were used for a contactless characterization of the b-Si photoelectronic properties and revealed the effects of the surface modification under various environmental conditions. Optimized b-Si production conditions allow very low $\sim 100 \mathrm{~cm} / \mathrm{s}$ surface recombination velocities on b-Si needles, which are critical for effective photovoltaic device operation. For modeling of optical properties and experimental response, a combination of the 
(a)

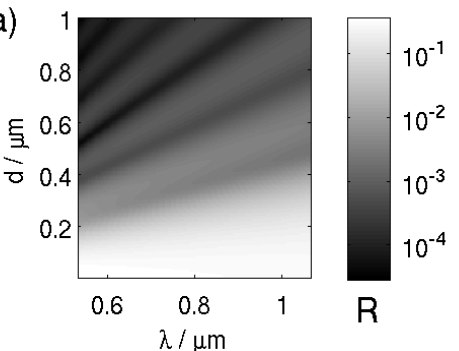

(b)

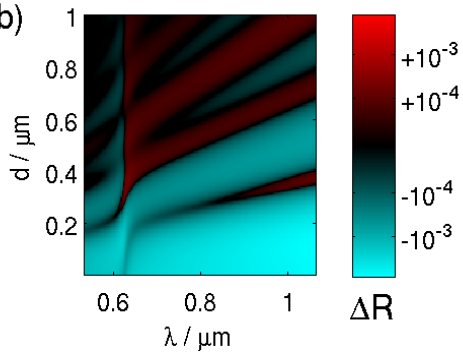

(c)

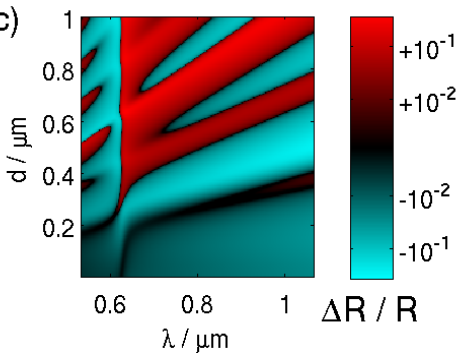

FIG. 5: Estimated differential reflection for a graded black Si layer (with linear conical profile) of varying thickness $d$, showing (a) unperturbed reflectance, (b) change in reflectance, and (c) relative reflectance change. Color represents the sign of the change (red higher, cyan lower). The calculation was performed using $532 \mathrm{~nm}$ pump and probe wavelengths as shown, for p-type $0.001 \Omega . c m ~ S i$. All of the higher resistivity results are similar, assuming that the backside reflection is not collected.

augmented Drude excited-carriers and graded effectivemedium models were employed. The revealed dependence of the differential reflectivity on probe wavelength (0.1 to $1 \mu \mathrm{m}$ ) and needle parameters (length in 0.5-1.1 $\mu \mathrm{m}$ range) has provided insights into tailoring the b-Si properties for sensor applications, based on refractive index modulation in the vicinity of the strong reflectivity dip.

The experimentally observed reflectivity of b-Si increased linearly with excitation intensity for pyramidal needles $(\Delta R>0)$, while it decreased for $\mathrm{Si}(\Delta R<$ $0)$, indicating excitation independent absorption. Also, some of the b-Si surfaces showed very low reflectivity of $\sim 0.01 \%$. Both of these properties can potentially be useful for designing sensors and engineering light harvesting systems.

\section{Experimental Section}

Black-Si samples Samples of b-Si were made from a single-side polished p-type $\langle 100\rangle$ orientation $\mathrm{Si}$ wafer (Atecom Ltd, Taiwan) with specific resistivity of 10 $20 \Omega$.cm, unless otherwise specified. Plasma dry etching for nano-texturing the Si surface was carried out in a flow of mixed $\mathrm{SF}_{6}: \mathrm{O}_{2}$ at controlled bias (dry reactive ion plasma etching - DRIE) and voltage of the inductivelycoupled plasma (ICP). The interplay between RIE and ICP controls the surface etching anisotropy together with the chamber pressure.

Batch No. 1 was made on a RIE-101iPH (SAMCO Inc.) etching tool capable of performing RIE and ICP-assisted RIE experiments. The $150 \mathrm{~W}$ ICP and $15 \mathrm{~W}$ (RIE) bias powers were typically used at a gas flow of $35 \mathrm{sccm}$ for $\mathrm{SF}_{6}$ and $45 \mathrm{sccm}$ for $\mathrm{O}_{2}$. The chamber pressure was $1 \mathrm{~Pa}$ and etching time was $15 \mathrm{~min}$. This resulted in pyramidally shaped pillars. This recipe was used for the majority of samples tested in the pump-probe experiments.

Batches No. 2 and 3 were fabricated in an Oxford PlasmaLab 100 ICP380 system, which allows a greater degree of structural control. Here the $\mathrm{SF}_{6}$ gas flow rate was $65 \mathrm{sccm}, \mathrm{O}_{2}$ gas flow rate $44 \mathrm{sccm}$, process pressure
35 mTorr, $100 \mathrm{~W}$ RIE power, $20{ }^{\circ} \mathrm{C}$ electrode temperature and 10 Torr He backside cooling pressure ${ }^{5}$. Nanopillars of aspect ratio up to $2.2 \pm 0.3$ were formed in $\sim 25$ min over the full area of 3 - and 4 -inch wafers under these conditions.

Magnetron sputtering (AXXIS, Kurt J. Lesker Ltd) was used for $\mathrm{Au}$ and $\mathrm{Pd}$ deposition with a well-controlled thickness for scanning electron microscope (SEM) inspection as well as for pump-probe experiments. Thickness of coating corresponds to that on a flat substrate. Transmission electron microscopy (TEM) analysis was carried out on a Jeol 2100F microscope equipped with a energy dispersive spectrometer (EDS) for surface chemical analysis.

The separation between b-Si needles followed a diffusional dependence on the etching time $x(t)=x(t=$ $5 \min ) \sqrt{t}$. For Batches No. 2 and 3 the average separation was $388 \pm 20 \mathrm{~nm}$ and $842 \pm 30 \mathrm{~nm}$, respectively (Fig. 1). For Batch No. 1, the separation was $\sim 300 \mathrm{~nm}$ and the pillars had a pyramidal shape of height $\sim 500 \mathrm{~nm}$, similar to Batch No. 2. Batches 1 and 2 had comparable pillar heights, but for Batch 3 the height was $\sim 4 \mu \mathrm{m}$ as the etching time was longer. For etching times above 0.5 $\mathrm{h}$ strong pillar height disorder sets in, leading to short wavelength light scattering ${ }^{11}$.

Ellipsometry measurements A Woollam V-Vase Ellipsometer was used to measure the ellipsometric reflection values of $\Psi$ and $\Delta$. To complement the ellipsometry measurements polarised angular dependent reflectance was also assessed using a Perkin Elmer Lambda 950 with a Universal Reflectance Accessory. Following this the measured data was fitted using a graded effective medium profile to model the geometrical profile of the random pyramidal structures via a spectrally wide best fit. The thickness of the oxide layer was also used as a fit parameter. Standard multilayer thin film calculations based on a graded effective medium ${ }^{28}$ were used for the needles. As the ellipsometry measurement is specular in nature, the backside reflectance of the $\mathrm{Si}$ wafer was neglected for the ellipsometric analysis (as the backside was un-polished). The collected data was fitted using the 
Woollam WVASE software with the optical model in order to estimate the refractive index and Si concentration profile through the thickness of the b-Si layer. Starting b-Si profile values were based on the SEM profile, i.e. needles on top of pyramids (Fig. 1). In addition to Si and void, a native silicon dioxide component was included to achieve a better fit. The profile parameters were allowed to vary until a good fit to experimental data was obtained (Batch No.1). The fit was relatively poor below $0.5 \mu \mathrm{m}$, which is expected because of scattering due to the feature size being comparable to the wavelength of light.

Pump-probe measurements Photo-excitation (pump) of the samples in reflectivity $D R \equiv \Delta R / R$ and transmittivity $D T \equiv \Delta T / T$ measurements was performed by $15 \mathrm{ps}$ duration laser pulses at $532 \mathrm{~nm}$ wavelength. The excitation intensity was varied from $I_{0} \approx 0.1 \mathrm{~mJ} / \mathrm{cm}^{2}$ to $\sim 5 \mathrm{~mJ} / \mathrm{cm}^{2}$, which generated an excess carrier (electron-hole pairs) density at the excited surface $\Delta N_{0}=\alpha_{e x} I_{0} / h \nu_{e x}$ in a range from $\sim 10^{18} \mathrm{~cm}^{-3}$ up to $\sim 10^{20} \mathrm{~cm}^{-3}$. The photoexcited carriers were distributed within a layer of thickness $d_{e x}=1 / \alpha_{e x} \sim 1 \mu \mathrm{m}$. Here $h \nu_{e x}=2.33 \mathrm{eV}$ is the photon energy at the excitation wavelength and the absorption coefficient $^{15} \alpha_{\text {ex }} \simeq 10^{4} \mathrm{~cm}^{-1}$.

An optically delayed (up to $4 \mathrm{~ns}$ ) 25 ps duration probe beam at $\lambda_{p r}=1064 \mathrm{~nm}$ wavelength $\left(h \nu_{p r}=1.18 \mathrm{eV}\right)$ was used to monitor the fast decay transients of the induced differential reflectivity $(D R)$ and transmittivity $(D T)$ decay kinetics. For reflectivity and transmittivity detection at $1064 \mathrm{~nm}$, filters with a blocking ratio of $10^{-6}$ were used to block the $532 \mathrm{~nm}$ excitation radiation (note that the excitation and probe paths were also different to exclude excitation radiation at the probe detectors).

Pump-probe model calculations The pump-probe response shown in Fig. 5 was estimated using a combination of an augmented Drude excited-carrier model and a graded effective-medium model similar to that em- ployed in the ellipsometry. The material model assumed that the absorption of pump photons (at $532 \mathrm{~nm}$ ) is proportional to the product of the optical intensity and the imaginary part of the refractive index and that all photons absorbed in the silicon were converted into carriers. Separate Drude terms were used with electron and hole mobilities based on a known unpumped impurity concentration $^{15}$. It was determined that a graded doping profile would not be important in most cases. A transfermatrix thin-film model was employed, with 100 layers sampling the graded profile. A linear conical structure was simulated as a quadratic change in Si concentration ( 0 at cover and 1 at substrate) in a vertically oriented uniaxial Bruggeman effective-medium model, with zero vertical depolarization. Other profiles were tested but the linear profile is the simplest assumption that is consistent with experimental micrographs. The intensity-dependent refractive-index profile was made self-consistent with the optical intensity profile, although this coupling was found to be unimportant in most cases. Backside reflection would contribute to scattering from the sample at the resistivities used in the experiment, but was omitted due to the experimental geometry that excluded diffuse reflectance contributions, and as confirmed by the linear pump-power dependence.

\section{Acknowledgements}

PŠ acknowledges support from Lithuanian Academy of Science via the Young Scientists Scholarship. SJ is grateful for support by ARC Discovery DP130101205 grant. IA is the recipient of an Australian Research Council Discovery Early Career Research Award (Project No. DE130100592). AG and MA are grateful for support by ARC Discovery DP140102003 grant.
* Electronic address: Patrik.Scajev@ff.vu.lt

$\dagger$ Electronic address: SJuodkazis@swin.edu.au

${ }^{1}$ E. P. Ivanova, J. Hasan, H. K. Webb, G. Gervinskas, S. Juodkazis, V. K. Truong, A. H. F. Wu, R. N. Lamb, V. Baulin, G. S. Watson, J. A. Watson, D. E. Mainwaring, and R. J. Crawford, "Bactericidal activity of nanostructured black silicon," Nature Commun. 4, 2838, 2013.

2 G. Gervinskas, G. Seniutinas, J. S. Hartley, S. Kandasamy, P. R. Stoddart, and S. Juodkazis, "Surface-enhanced Raman scattering sensing on black silicon," Annalen der Physik 525(12), 907 - 914, 2013.

3 G. Seniutinas, G. Gervinskas, R. Verma, B. D. Gupta, F. Lapierre, P. R. Stoddart, F. Clark, S. L. McArthur, and S. Juodkazis, "Versatile SERS sensing based on black silicon," Optics Express 23(5), 6763 - 6772, 2015.

${ }^{4}$ R. Komatsu, A. Balčytis, G. Seniutinas, T. Yamamura, Y. Nishijima, and S. Juodkazis, "Plasmonic photothermoelectric energy converter with black-Si absorber,"
Solar Energy Materials Solar Cells 143, 72 - 77, 2015.

5 A. Žukauskas, M. Malinauskas, A. Kadys, G. Gervinskas, G. Seniutinas, S. Kandasamy, and S. Juodkazis, "Black silicon: substrate for laser 3D micro/nano-polymerization," Optics Express 21(6), 6901-6909, 2013.

${ }^{6}$ J. Oh, H.-C. Yuan, and H. M. Branz, "An 18.2

$\%$ efficient black-silicon solar cell achieved through control of carrier recombination in nanostructures," Nature Nanotechn. 7, 743-748, 2012.

7 K.-C. Park, H. J. Choi, C.-H. Chang, R. E. Cohen, G. H. McKinley, and G. Barbastathis, "Nanotextured silica surfaces with robust superhydrophobicity and omnidirectional broadband supertransmissivity," ACS Nano 5(5), 37893799, 2012.

${ }^{8}$ P. R. Stoddart, P. J. Cadusch, T. M. Boyce, R. M. Erasmus, and J. D. Comins, "Optical properties of chitin: surface-enhanced raman scattering substrates based on antireflection structures on cicada wings," Nanotechnol- 
ogy 17, 680-686, 2006.

${ }^{9}$ K. Juodkazis, J. Juodkazytè, B. Šebeka, I. Savickaja, and S. Juodkazis, "Photoelectrochemistry of silicon in HF solution," J. Solid State Electrochem. 17(8), 2269-2276, 2013.

10 S. Eyderman, S. John, and A. Deinega, "Solar light trapping in slanted conical-pore photonic crystals: Beyond statistical ray trapping," J. Appl. Phys. 113, 154315, 2013.

11 G. Gervinskas, G. Seniutinas, J. S. Hartley, S. Kandasamy, P. R. Stoddart, N. F. Fahim, and S. Juodkazis, "Surfaceenhanced Raman scattering sensing on black silicon," $A n$ nalen der Physik 525(12), 907-914, 2013.

12 A. J. Sabbah and D. M. Riffe, "Femtosecond pump-probe reflectivity study of silicon carrier dynamics," Phys. Rev. B 66, 165217, 2002.

13 G. Vuye, S. Fisson, V. Nguyen Van, Y. Wang, J. Rivory and F. Abelés, "Temperature dependence of the dielectric function of silicon using in situ spectroscopic ellipsometry," Thin Solid Films 233, 166-170, 1993.

${ }^{14}$ P. Ščajev, "Application of excite-probe techniques for determination of surface, bulk and nonlinear recombination rates in cubic SiC," Mater. Sci. Eng. B 185, 37-44, 2014.

15 S. M. Sze, Physics of Semiconductor Devices, John Wiley and Sons, New York, 1981

16 M. Cardona and F. H. Pollak, "Energy-band structure of germanium and silicon," Phys. Rev. B 142, 530-543, 1966.

17 B. J. Frey, D. B. Leviton, and T. J. Madison, "Temperature-dependent refractive index of silicon and germanium," in Optomechanical Technologies for Astronomy, E. Atad-Ettedgui, J. Antebi, and D. Lemke, eds., p. 62732J, Proc. SPIE vol. 6273, 2006.

18 P. Ščajev and K. Jarašiūnas, "Application of a timeresolved four-wave mixing technique for the determination of thermal properties of $4 \mathrm{H}-\mathrm{SiC}$ crystals," J. Phys. D: Appl. Phys. 42, 055413, 2009.
19 P. Flubacher, A. J. Leadbetter, and J. A. Morrison, "The heat capacity of pure silicon and germanium and properties of their vibrational frequency spectra," Phil. Mag. 4(39), 273-294, 1959

20 J. Linnros, "Carrier lifetime measurements using free carrier absorption transients. i. principle and injection dependence," J. Appl. Phys. 84, 275-283, 1998.

21 C. Klingshirn, Semiconductor Optics, Springer-Verlag, Berlin, 2nd ed., 2005.

22 P. Grivickas, Optical Studies of Carrier Transport and Fundamental Absorption in $4 \mathrm{H}-\mathrm{SiC}$ and Si. PhD thesis, Royal Institute of Technology, Sweeden, 2004.

${ }^{23}$ P. Ščajev, A. Usikov, V. Soukhoveev, R. Aleksiejūnas, and K. Jarašiūnas, "Diffusion-limited nonradiative recombination at extended defects in hydride vapor phase epitaxy GaN layers," Appl. Phys. Lett. 98, 202105, 2011.

24 S. Glunz, R. Preu, and D. Biro, Comprehensive Renewable Energy, vol. 1, ch. Crystalline Silicon Solar Cells - Stateof-the-Art and Future Developments. Elsevier, 2012.

${ }^{25}$ S. Kumar, P. K. Singh, G. S. Chilana, and S. R. Dhariwal, "Generation and recombination lifetime measurement in silicon wafers using impedance spectroscopy," Semicond. Sci. Technol. 24, 095001, 2009.

26 R. D. Westbrook, Lifetime Factors in Silicon, Baltimore, 1980.

27 J. Dziewior and W. Schmid, "Auger coefficients for highly doped and highly excited silicon," Appl. Phys. Lett. 31, 346-348, 1977.

28 P. G. Snyder, Y. M. Xiong, J. A. Woollam, G. A. A. Jumaily, and F. J. Gagliardi, "Graded refractive index silicon oxynitride thin film characterized by spectroscopic ellipsometry,", J. Vac. Sci. Technol. A10, 1462, 1992. 